This item was submitted to Loughborough's Research Repository by the author.

Items in Figshare are protected by copyright, with all rights reserved, unless otherwise indicated.

\title{
Sharp spectral bounds for complex perturbations of the indefinite Laplacian
}

PLEASE CITE THE PUBLISHED VERSION

https://doi.org/10.1016/j.jfa.2020.108804

PUBLISHER

Elsevier

VERSION

AM (Accepted Manuscript)

PUBLISHER STATEMENT

This paper was accepted for publication in the journal Journal of Functional Analysis and the definitive published version is available at https://doi.org/10.1016/j.jfa.2020.108804

\section{LICENCE}

CC BY-NC-ND 4.0

\section{REPOSITORY RECORD}

Cuenin, Jean-Claude, and OO Ibrogimov. 2020. "Sharp Spectral Bounds for Complex Perturbations of the Indefinite Laplacian”. Loughborough University. https://hdl.handle.net/2134/13222184.v1. 


\title{
SHARP SPECTRAL BOUNDS FOR COMPLEX PERTURBATIONS OF THE INDEFINITE LAPLACIAN
}

\author{
JEAN-CLAUDE CUENIN AND ORIF O. IBROGIMOV
}

\begin{abstract}
We derive quantitative bounds for eigenvalues of complex perturbations of the indefinite Laplacian on the real line. Our results substantially improve existing results even for real potentials. For $L^{1}$-potentials, we obtain optimal spectral enclosures which accommodate also embedded eigenvalues, while our result for $L^{p}$-potentials yield sharp spectral bounds on the imaginary parts of eigenvalues of the perturbed operator for all $p \in[1, \infty)$. The sharpness of the results are demonstrated by means of explicit examples.
\end{abstract}

\section{INTRODUCTION AND MAIN RESULtS}

Spectral estimates for non-self-adjoint Schrödinger operators and related questions have been a very active area of mathematical research recently. By now several robust methods have been developed and the corresponding literature is extensive. To name a few, we mention $99,16,19,22,28,31,33,36,36,38,42$ and also [17, 20, 21, for related recent results obtained in an abstract operator theoretic setting.

To goal of this paper is to establish sharp spectral estimates for the operator

$$
H_{V}:=\operatorname{sgn}(x)\left(-\partial_{x}^{2}+V\right) \quad \text { in } \quad L^{2}(\mathbb{R}) .
$$

The unperturbed operator $H_{0}$ is not symmetric in the Hilbert space $L^{2}(\mathbb{R})$ due to the sign change of the weight function; it can be interpreted as a self-adjoint operator with respect to the Krein space inner product $(\operatorname{sgn} \cdot, \cdot)$ in $L^{2}(\mathbb{R})$. The sum in 1.1 is defined in the form sense (see Section 2 for details)

Unlike its definite counterpart (i.e. without the sign function), the existing literature on eigenvalue bounds for operators of type (1.1) seems to be much more sparse even in the one-dimensional setting with real potentials, see e.g. [4, 6, 8, and a number of interesting questions remain open. One of them is a conjecture of Behrndt in [3] according to which the eigenvalues of singular indefinite Sturm-Liouville operators (i.e. (1.1) with real-valued $V$ ) accumulate to the real axis whenever the eigenvalues of the corresponding definite SturmLiouville operator accumulate to the bottom of the essential spectrum from below. So far the conjecture of Behrndt has been confirmed only for a particular family of (shifted Coulomb type) potentials in 39 .

The present paper is partially motivated by the recent work [7, where the following (non optimal) indefinite analogue of the celebrated result of [1] was shown: every non-real eigenvalue $\lambda$ of the singular indefinite Sturm-Liouville operator with a real-valued potential $V \in L^{1}(\mathbb{R})$ obeys the estimate

$$
|\lambda|^{1 / 2} \leq\|V\|_{1}
$$

Date: August 6, 2020. 
Our standing assumption is that there exist numbers $a \in(0,1)$ and $b \in \mathbb{R}$ such that, for all $\psi \in H^{1}(\mathbb{R})$,

$$
\int_{\mathbb{R}}|V||\psi|^{2} \leq a \int_{\mathbb{R}}\left|\psi^{\prime}\right|^{2}+b \int_{\mathbb{R}}|\psi|^{2}
$$

In particular, potentials $V \in L^{p}(\mathbb{R})$ are covered by this hypothesis for all $p \in[1, \infty]$, see Section 2 for more details. Furthermore, in the same way, it is also possible to proceed in a greater generality and give a meaning to the distributional Dirac delta potential $\delta$, which is explicitly solvable. Putting $\|\delta\|_{L^{1}(\mathbb{R})}:=1$ by convention, Theorem 1 remains valid in this more general setting. The feature of our results are that we work with complex potentials of minimal regularity, our bounds are quantitative and substantially improve existing results. Moreover, for $L^{1}$-potentials our spectral enclosures are sharp and we cover embedded eigenvalues, too.

Throughout the paper we denote by $\|\cdot\|_{p}$ the standard $L^{p}$-norm for $p \in[1, \infty]$. Our first result quantitatively improves the known bound (1.2) of [7] and holds also for possibly embedded eigenvalues.

Theorem 1. For $V \in L^{1}(\mathbb{R})$, every eigenvalue $\lambda$ of $H_{V}$ satisfies

$$
\sqrt{2}|\lambda| \leq \sqrt{|\lambda|+|\Re(\lambda)|}\|V\|_{1} .
$$

Remark 1. (i) The bound in (1.4) is sharp in the sense that, for any $Q>0$ and any point $\lambda \in \mathbb{C} \backslash \mathbb{R}$ which fulfills the equation

$$
2|\lambda|^{2}=(|\lambda|+|\Re(\lambda)|) Q^{2},
$$

there exists $V \in L^{1}(\mathbb{R})$ such that $Q=\|V\|_{1}$ and $\lambda$ is an eigenvalue of the corresponding operator $H_{V}$, see Section 5.2. This means that every boundary point of the spectral enclosure corresponding to (1.4), with the exception of points located in the real line, is an eigenvalue of $H_{V}$ for some $L^{1}$-potential $V$. Hence the obtained spectral enclosure cannot be squeezed any further.

(ii) It readily follows from Theorem 1 that the intervals $\left(-\infty,\|V\|_{1}^{2}\right)$ and $\left(\|V\|_{1}^{2}, \infty\right)$ are free of embedded eigenvalues of $H_{V}$ for $V \in L^{1}(\mathbb{R})$.

(iii) In view of the elementary inequality $|\Re(\lambda)| \leq|\lambda|$, it is obvious that the bound 1.4 provides a strictly tighter spectral enclosure than $(1.2)$.

Corollary 1. For $V \in L^{1}(\mathbb{R})$, every eigenvalue $\lambda$ of $H_{V}$ satisfies the sharp bound

$$
|\Im(\lambda)| \leq \frac{3 \sqrt{3}}{8}\|V\|_{1}^{2}
$$

The spectral enclosure corresponding to 1.4 is a compact set, which is symmetric with respect to both the real and the imaginary axes. The geometry of its boundary is quite easy to understand. We refer to Figure 1 for the plots of the boundary curves corresponding to (1.2) and (1.4) with $\|V\|_{1}=1$.

Our next result provides a spectral estimate in terms of the $L^{p}$-norm of the potential for $p \in(1, \infty)$. The proof interpolates the bound of Theorem 1 with the standard operator norm bound for the resolvent of a self-adjoint operator. The strategy was first used in [27].

Theorem 2. Let $p \in(1, \infty)$. For $V \in L^{p}(\mathbb{R})$, every eigenvalue $\lambda$ of $H_{V}$ satisfies

$$
2^{\frac{3}{2 p}-1}|\lambda|^{\frac{1}{p}}|\Im(\lambda)|^{1-\frac{1}{p}} \leq(|\lambda|+|\Re(\lambda)|)^{\frac{1}{2 p}}\|V\|_{p} .
$$



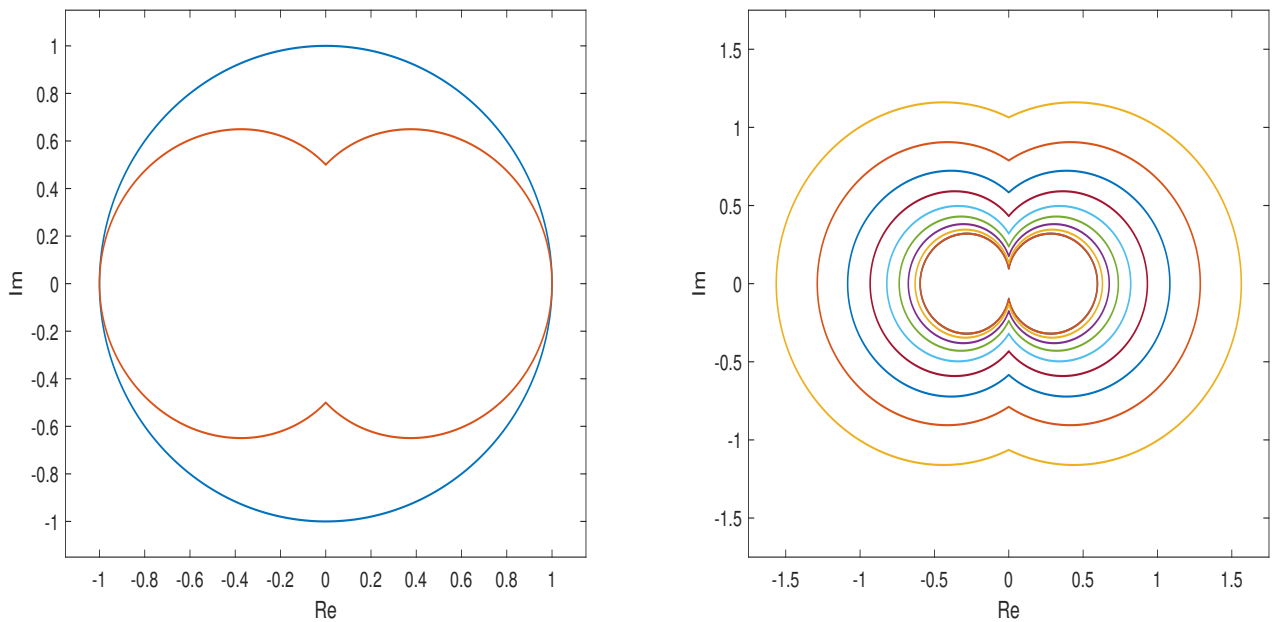

Figure 1. The blue circle and the red curve on the left correspond to the boundary curves of the spectral enclosures respectively given by 1.2 and 1.4 for $\|V\|_{L^{1}(\mathbb{R})}=1$. The picture on the right illustrates plots of the expanding boundary curves corresponding to the spectral enclosure (1.4) for $\|V\|_{1}=$ $(1.35)^{j} / 7, j=1,2, \ldots, 9$.

Remark 2. (i) The bound 1.6 trivially holds for embedded eigenvalues. Letting $p \searrow$ 1 in 1.6 , one arrives at the bound 1.4. An analogous version of the bound 1.6 for the case $p=\infty$ is very easy to derive and reads as

$$
|\Im(\lambda)| \leq 2\|V\|_{\infty} \text {. }
$$

(ii) Using the elementary inequalities $|\Re(\lambda)| \leq|\lambda|$ and $|\Im(\lambda)| \leq|\lambda|$, one can easily read off from $(1.6)$ that the following rough bound holds

$$
|\Im(\lambda)| \leq 2^{\frac{2 p-2}{2 p-1}}\|V\|_{p}^{\frac{2 p}{2 p-1}} .
$$

This improves the recent result in $[40]$ where, for real-valued $V \in L^{p}(\mathbb{R})$ with $p \geq 2$, the estimate (1.7) was shown to hold with a constant factor $C(p)$ such that $C(p)>2^{\frac{2 p-2}{2 p-1}}$. While the result of [40] is a considerable improvement of the analogous result of 8 , the function $C(p)$ therein is a strictly decreasing function of the parameter $p$ with the limit equal to $2+\sqrt{2}$ at infinity.

As a matter of fact, by an optimization trick under the constraint (1.6), one can substantially improve the bound (1.7), thus generalizing the sharp bound 1.5$)$ for $L^{p}$-potentials for all $p \in(1, \infty)$.

Corollary 2. Let $p \in(1, \infty]$. For $V \in L^{p}(\mathbb{R})$, every eigenvalue $\lambda$ of $H_{V}$ satisfies the bound

$$
|\Im(\lambda)| \leq 2\left(\frac{3 \sqrt{3}}{16}\right)^{\frac{1}{2 p-1}}\|V\|_{p}^{\frac{2 p}{2 p-1}} .
$$

Below we provide various plots of the expanding boundary curves corresponding to the spectral enclosure from Theorem 2 .

The next result concerns with spectral bounds for $L^{p}$-potentials again. This time, however, it is given in terms of the $L^{p}$-sizes of the restrictions to the intervals $(0, \infty)$ and $(-\infty, 0)$ 


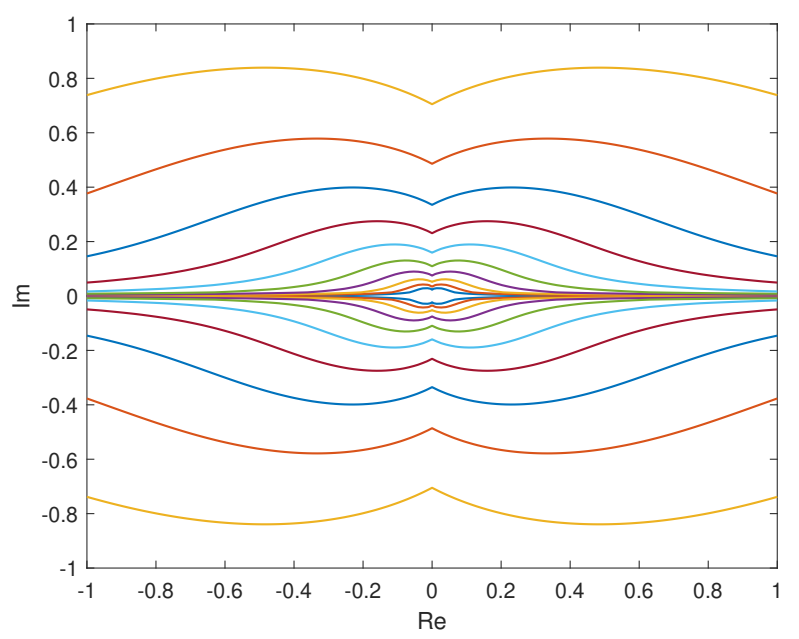

Figure 2. The plots of the expanding boundary curves corresponding to the spectral enclosure from Theorem 2 for $p=1.25$ and $\|V\|_{p}=(1.25)^{j} / 10$, $j=1,2, \ldots, 10$.

of the potential. In the sequel, for $p \in(1, \infty)$, we denote by $\|V\|_{p, \pm}$ the standard $L^{p}$-norms over the intervals $(0, \infty)$ and $(-\infty, 0)$, respectively.

Theorem 3. Let $p \in(1, \infty)$. For $V \in L^{p}(\mathbb{R})$, every non-real eigenvalue of $H_{V}$ satisfies

$\sqrt{2}\left(\frac{p}{p-1}\right)^{1-\frac{1}{p}} \sqrt{|\lambda|} \leq \max \left\{\frac{\|V\|_{p,-}}{|\Re(\sqrt{\lambda})|^{1-\frac{1}{p}}}+\frac{\sqrt{2}\|V\|_{p,+}}{|\Im(\sqrt{\lambda})|^{1-\frac{1}{p}}}, \frac{\sqrt{2}\|V\|_{p,-}}{|\Re(\sqrt{\lambda})|^{1-\frac{1}{p}}}+\frac{\|V\|_{p,+}}{|\Im(\sqrt{\lambda})|^{1-\frac{1}{p}}}\right\}$.

Remark 3. (i) In general, neither of the spectral bounds of Theorems 2 and 3 is better than the other. Numerical experiments, however, indicate that the one of Theorem 2 is better than that of Theorem 3 for sufficiently small values of $p>1$, while the opposite is true for sufficiently large values of $p$.

(ii) Unlike that of Theorem 1 the spectral enclosures of Theorems 2 and 3 are non-compact sets, yet being symmetric with respect to both the real and the imaginary axes.

Note that the bounds (1.2), (1.4)-1.6) are scale-invariant. More precisely, if $U_{\rho}$ is the unitary operator $U_{\rho} f(x)=\rho^{-1 / 2} f(x / \rho), \rho>0$, then

$$
U_{\rho}^{*} H_{V} U_{\rho}=\rho^{-2} \operatorname{sgn}(x)\left(-\partial_{x}^{2}+V_{\rho}\right), \quad V_{\rho}(x)=\rho^{2} V(\rho x),
$$

has the same eigenvalues as $H_{V}$. Hence,

$$
\lambda \in \sigma_{\mathrm{p}}\left(H_{V}\right) \Longleftrightarrow \rho^{2} \lambda \in \sigma_{\mathrm{p}}\left(H_{V_{\rho}}\right) .
$$

Since $\left\|V_{\rho}\right\|_{p}=\rho^{2-1 / p}\|V\|_{p}$ and $|\cdot|, \Im(\cdot), \Re(\cdot)$ are homogeneous of degree one, applying any of the inequalities 1.2, 1.4 1.6 to $H_{V_{\rho}}$ instead of $H_{V}$ does not change the outcome. ity

Next, by a Wigner-von Neumann type example we show that the scale-invariant inequal-

$$
|\lambda|^{p-\frac{1}{2}} \leq C\|V\|_{p}^{p}
$$


which holds for $p=1$ by 1.2 , cannot hold for $p>1$. The idea to consider complex-valued Wigner-von Neumann potentials in the context of spectral estimates for non-self-adjoint operators is due to Frank and Simon [30].

Theorem 4. Let $\lambda>0$ be arbitrary. There exists a positive constant $C=C(\lambda)$ and a sequence of potentials $V_{n}: \mathbb{R} \rightarrow \mathbb{R}$ such that, for all $n, \lambda$ is an eigenvalue of $H_{V_{n}}$ and

$$
\left|V_{n}(x)\right| \leq \frac{C}{n+|x|}, \quad x \in \mathbb{R} .
$$

In particular, for any $p>1$, we have

$$
\lim _{n \rightarrow \infty}\left\|V_{n}\right\|_{p}=0 .
$$

We now modify the above result to produce a non-real eigenvalue instead of an embedded one, showing that the scale-invariant inequality (1.11) also fails in this case. Additionally, we demonstrate that the exponents in 1.6 cannot be improved.

Theorem 5. Given $\varepsilon>0$ sufficiently small and $\mu>0$, there exists a potential $V=$ $V(\varepsilon, \mu) \in L^{\infty}(\mathbb{R})$ with compact support and such that $H_{V}$ has eigenvalue $\lambda=\lambda(\varepsilon, \mu)$ with $\Re(\lambda)=\mu(1+\mathcal{O}(\varepsilon)), \Im(\lambda)=\mu \varepsilon\left(2+\mathcal{O}\left(\varepsilon^{2}\right)\right)$, and for every $p \geq 1$,

$$
\|V(\varepsilon, \mu)\|_{p} \approx|\mu|^{1-\frac{1}{2 p}} \varepsilon^{1-\frac{1}{p}}|\ln \varepsilon|^{\frac{1}{p}} .
$$

In particular, for $p>1$, we have

$$
\lim _{\varepsilon \rightarrow 0}\|V(\varepsilon, \mu)\|_{p}=0
$$

Remark 4. Theorem 5 can be reformulated in the following way: Consider the sector

$$
\Sigma_{\varepsilon_{0}}:=\left\{\lambda \in \mathbb{C}:|\Im(\lambda)| \leq \varepsilon_{0}|\Re(\lambda)|\right\},
$$

where $\varepsilon_{0}$ is sufficiently small but fixed. Then, given $\lambda$ in the intersection of $\Sigma_{\varepsilon_{0}}$ with the first quadrant, there exists a potential $V=V(\lambda) \in L_{c}^{\infty}(\mathbb{R})$ such that $H_{V}$ has eigenvalue $\lambda$ and, for every $p \geq 1$,

$$
\|V(\lambda)\|_{p} \approx|\lambda|^{\frac{1}{2 p}}|\Im(\lambda)|^{1-\frac{1}{p}}\left|\ln \left(\frac{|\Im(\lambda)|}{|\lambda|}\right)\right|^{\frac{1}{p}} .
$$

This is easily seen by observing that we can find $\lambda=\lambda(\varepsilon, \mu)$ as in the theorem with

$$
\mu=\Re(\lambda)\left(1+\mathcal{O}\left(\varepsilon_{0}\right)\right), \quad \varepsilon=\frac{\Im(\lambda)}{2 \Re(\lambda)}\left(1+\mathcal{O}\left(\varepsilon_{0}\right)\right), \quad|\lambda|=\Re(\lambda)\left(1+\mathcal{O}\left(\varepsilon_{0}\right)\right) .
$$

It is also clear that the assumption of $\lambda$ lying in the first quadrant can be omitted.

In the next result we study the weak coupling limit, i.e. we replace $V$ by $\varepsilon V$ in $H_{V}$ and establish existence, uniqueness and asymptotics of an eigenvalue $\lambda(\varepsilon)$ as $\varepsilon \rightarrow 0^{+}$. This will yield another confirmation that the spectral bound 1.4 is sharp. In the following we set

$$
v_{+}=\int_{0}^{\infty} V(x) \mathrm{d} x, \quad v_{-}=\int_{-\infty}^{0} V(x) \mathrm{d} x, \quad v_{\text {sgn }}=\int_{-\infty}^{\infty} \operatorname{sgn}(x) V(x) \mathrm{d} x .
$$

Theorem 6. Assume that $\Re\left(v_{\text {sgn }}\right)+\Im\left(v_{\text {sgn }}\right)<0$ and $\Re\left(v_{\text {sgn }}\right)<\Im\left(v_{\text {sgn }}\right)$. Then, for all sufficiently small $\varepsilon>0$, there exists a unique eigenvalue $\lambda(\varepsilon) \in \mathbb{C}^{+}$of $H_{\varepsilon V}$ satisfying

$$
\lambda(\varepsilon)=\frac{\varepsilon^{2}}{(1-i)^{2}} v_{\mathrm{sgn}}^{2}+o\left(\varepsilon^{2}\right), \quad \varepsilon \rightarrow 0^{+} .
$$


Our next result is a particular case of a Lieb-Thirring type bound. This is an analogue to a special case of [27, Thm 1.3] for the definite Schrödinger operator. In fact, the conclusions of [27, Thm 1.2] and 27, Thm 1.3] in the case $d=1$ there continue to hold for the indefinite operator $H_{V}$ considered here.

Theorem 7. For $V \in L^{1}(\mathbb{R})$, we have

$$
\sum_{j}\left|\Im\left(\lambda_{j}\right)\right| \leq C\|V\|_{1}^{2}
$$

where $\lambda_{j}$ are the eigenvalues of $H_{V}$ repeated according to their algebraic multiplicities.

We use the method of [29] to bound the number of eigenvalues of $H_{V}$ in terms of an exponentially weighted $L^{1}$ norm of $V$ as follows.

Theorem 8. The number of eigenvalues $N(V)$ of $H_{V}$, counting algebraic multiplicities, satisfies, for any $\varepsilon>0$,

$$
N(V) \leq \frac{1}{\varepsilon^{2}}\left(\int_{\mathbb{R}} \mathrm{e}^{\varepsilon|x|}|V(x)| \mathrm{d} x\right)^{2} .
$$

The outline of the paper is as follows. In Section 2 , we rigorously introduce the perturbed operator (1.1) using form methods. In Section 3. we derive a sharp estimate on the integral kernel of the free resolvent as well as a bound on a Krein type resolvent. In Section 4, we establish a limiting absorption principle; this can be viewed as a one-sided conventional Birman-Schwinger principle which holds also for embedded eigenvalues. The proofs of Theorems 1, 8 and Corollaries 1, 2 are given in Section 5.

\section{Definition of the operator $H_{V}$}

Let $T_{0}$ be the the self-adjoint operator in $L^{2}(\mathbb{R})$ associated with the quadratic form

$$
t_{0}[\psi]:=\int_{\mathbb{R}}\left|\psi^{\prime}\right|^{2}, \quad \mathrm{D}\left(t_{0}\right):=H^{1}(\mathbb{R}) .
$$

One has $\mathrm{D}\left(T_{0}\right)=H^{2}(\mathbb{R})$ and $T_{0}=-\partial_{x}^{2}$. The spectrum of $T_{0}$ is purely absolutely continuous and coincides with the semi-axis $[0,+\infty)$.

Let $v$ be a quadratic form in $L^{2}(\mathbb{R})$, which is relatively bounded with respect to $t_{0}$ with the relative bound less than one. That is, $\mathrm{D}(v) \supset H^{1}(\mathbb{R})$ and there exist numbers $a \in(0,1)$ and $b \in \mathbb{R}$ such that, for all $\psi \in H^{1}(\mathbb{R})$,

$$
|v[\psi]| \leq a \int_{\mathbb{R}}\left|\psi^{\prime}\right|^{2}+b \int_{\mathbb{R}}|\psi|^{2} .
$$

Then the sum $t_{V}:=t_{0}+v$ is a closed sectorial form with $\mathrm{D}\left(t_{V}\right)=H^{1}(\mathbb{R})$, which gives rise to an m-sectorial operator $T_{V}$ in $L^{2}(\mathbb{R})$ via the representation theorem (cf. 35 , Thm. VI.2.1]).

For example, if $V \in L_{\text {loc }}^{1}(\mathbb{R})$ is such that

$$
v[\psi]:=\int_{\mathbb{R}} V|\psi|^{2}, \quad \mathrm{D}(v):=\left\{\psi \in L^{2}(\mathbb{R}): \int_{\mathbb{R}}|V||\psi|^{2}<\infty\right\},
$$

verifies 2.2 (which coincides with 1.3 in this case), then we write $T_{V}=T_{0} \dot{+} V$.

Let us now discuss sufficient conditions which guarantee 2.2.

By the Sobolev embedding theorem ( 2 , Thm. 5.4]), every function $\psi \in H^{1}(\mathbb{R})$ is bounded and continuous. More specifically ( $c f$. [41, Theorem IX.28]), for any positive $\alpha$ there is $\beta \in \mathbb{R}$ such that, for all $\psi \in H^{1}(\mathbb{R})$,

$$
\|\psi\|_{\infty} \leq \alpha\left\|\psi^{\prime}\right\|_{2}+\beta\|\psi\|_{2}
$$


Consequently, any potential $V \in L^{1}(\mathbb{R})+L^{\infty}(\mathbb{R})$ satisfies $(1.3)$ with the relative bound equal to zero (i.e. $a$ can be chosen arbitrarily small).

\section{Free Resolvent and a Krein type Resolvent formula}

For real-valued $L^{p}$-potentials, the perturbed operator can be viewed as a self-adjoint operator in the Krein space with the indefinite inner product $(\operatorname{sgn} \cdot, \cdot)$. Consequently, the spectrum of the perturbed operator is symmetric with respect to the real axis, see e.g. 18 , 34. Certainly, this property no longer holds for general complex-valued potentials. As it was mentioned in the Introduction, it turns out, however, that our spectral enclosures are symmetric with respect to the both the real and the imaginary axes. Unless specified otherwise, for the rest of the paper we will work with a fixed spectral parameter $\lambda$ from the upper half-plane $\mathbb{C}^{+}$since the analysis of the lower part of the spectrum of $H_{V}$ is identical to that of the part in the upper half-plane.

3.1. Free resolvent. In the Hilbert space $L^{2}(\mathbb{R})$, let us consider the unperturbed operator

$$
H_{0}:=\operatorname{sgn}(x)\left(-\partial_{x}^{2}\right), \quad \mathrm{D}\left(H_{0}\right):=H^{2}(\mathbb{R}) .
$$

The spectrum of $H_{0}$ is continuous and coincides with $\mathbb{R}$. For $\lambda \in \mathbb{C}^{+}$, we denote by $G_{\lambda}$ the Green's function of $H_{0}-\lambda$, i.e. the integral kernel of the free resolvent $\left(H_{0}-\lambda\right)^{-1}$, which can be determined explicitly using the well-known form of the Green's function for the definite counterpart of $H_{0}$. In fact, we have $\left.\right|^{1}$

$$
G_{\lambda}(x, y)=\frac{1}{2 \alpha \sqrt{\lambda}} \begin{cases}\alpha e^{i \sqrt{\lambda}(x+y)}+\bar{\alpha} e^{i \sqrt{\lambda}|x-y|} & x \geq 0, y \geq 0, \\ -e^{\sqrt{\lambda}(i x+y)} & x \geq 0, y<0, \\ e^{\sqrt{\lambda}(x+i y)} & x<0, y \geq 0, \\ -\bar{\alpha} e^{\sqrt{\lambda}(x+y)}-\alpha e^{-\sqrt{\lambda}|x-y|} & x<0, y<0,\end{cases}
$$

where $\alpha:=\frac{1-i}{2}$ (see also $[7]$ ). Observe that, for all non-zero $x, y$, we have

$$
\operatorname{sgn}(x) G_{\lambda}(x, y)=\operatorname{sgn}(y) G_{\lambda}(y, x) .
$$

The pointwise estimate of the Green's function obtained in the next lemma plays a crucial role in establishing Theorem 1 .

Lemma 1. Let $\lambda \in \mathbb{C}^{+}$. The Green's function in 3.1) obeys the sharp pointwise estimate

$$
\left|G_{\lambda}(x, y)\right|^{2} \leq \frac{1}{2|\lambda|}+\frac{|\Re(\lambda)|}{2|\lambda|^{2}} .
$$

Proof. Let $a:=\Re(\sqrt{\lambda})>0$ and $b:=\Im(\sqrt{\lambda})>0$. We distinguish the four cases.

Case $x \geq 0, y \geq 0$. In view of 3.2 , there is no loss of generality in assuming that $x \geq y$. Then elementary calculations yield

$$
\left|G_{\lambda}(x, y)\right|^{2}=\frac{1}{4|\lambda|} \Phi(x, y),
$$

where

$$
\Phi(x, y):=e^{-2 b(x+y)}+e^{-2 b(x-y)}+2 e^{-2 b x} \sin (2 a y) .
$$

We fix $y \geq 0$ and consider $\Phi$ as a function of the variable $x$ on the interval $[y, \infty)$. Since $\Phi$ is non-negative for obvious reasons, and

$$
\frac{\partial}{\partial x} \Phi(x, y)=-2 b \Phi(x, y) \leq 0,
$$

\footnotetext{
${ }^{1}$ Here and in the sequel we choose the branch of the square root with $\Im(\sqrt{\lambda})>0, \Re(\sqrt{\lambda})>0\left(\lambda \in \mathbb{C}^{+}\right)$.
} 
we conclude that $\Phi(\cdot, y)$ is non-increasing on $[y, \infty)$. Therefore,

$$
\Phi(x, y) \leq \Phi(y, y)=1+e^{-4 b y}+2 e^{-2 b y} \sin (2 a y)=: \varphi(y) .
$$

In view of the identities $\Re(\lambda)=a^{2}-b^{2}$ and $\Im(\lambda)=2 a b$, it thus suffices to show that

$$
\max _{y \geq 0} \varphi(y) \leq 2\left(1+\frac{\left|a^{2}-b^{2}\right|}{a^{2}+b^{2}}\right) .
$$

It is not difficult to check that

$$
\varphi^{\prime}(y)=0 \quad \Longleftrightarrow \quad e^{-2 b y}=\frac{a}{b} \cos (2 a y)-\sin (2 a y) .
$$

In view of this, elementary calculations show that, if $y_{0} \geq 0$ is a critical point of $\varphi$, then it must hold that

$$
\varphi\left(y_{0}\right)=\left(1+\frac{a^{2}}{b^{2}}\right) \cos ^{2}\left(2 a y_{0}\right) .
$$

Let $t_{0}:=\cos \left(2 a y_{0}\right)$. If $t_{0} \leq 0$, then 3.9 implies that $\sin \left(2 a y_{0}\right)<0$. Then $\varphi\left(y_{0}\right)<$ $1+e^{-4 b y_{0}} \leq 2$ and 3.8 holds. If $b \geq a t_{0}$, then $\varphi\left(y_{0}\right) \leq 1+t_{0}^{2} \leq 2$ and 3.8 follows. If $\sin \left(2 a y_{0}\right)<0$, then again $\varphi\left(y_{0}\right)<2$, implying (3.8). So let us assume that $t_{0}>0, a t_{0}>b$ and $\sin \left(2 a y_{0}\right) \geq 0$. Then $\sin \left(2 a y_{0}\right)=\sqrt{1-t_{0}^{2}}$ and $(3.9)$ implies that

$$
0<\frac{a t_{0}}{b}-1 \leq \sqrt{1-t_{0}^{2}} .
$$

Squaring both sides of the latter, we conclude

$$
0<t_{0} \leq \frac{2 a b}{a^{2}+b^{2}} .
$$

On the other hand, the condition $a t_{0}>b$ together with $(3.12)$ imply that $b \leq a$. Therefore, in this case, we have

$$
\varphi\left(y_{0}\right)=t_{0}^{2}\left(1+\frac{a^{2}}{b^{2}}\right) \leq \frac{4 a^{2}}{a^{2}+b^{2}}=2\left(1+\frac{\left|a^{2}-b^{2}\right|}{a^{2}+b^{2}}\right) .
$$

Finally, by noticing that $\varphi(y) \rightarrow 2$ as $y \downarrow 0$ and that $\varphi(y) \rightarrow 1$ as $y \uparrow+\infty$, and summing up the above observations, we conclude 3.8.

Case $x \geq 0, y<0$. In this case, we have

$$
\left|G_{\lambda}(x, y)\right|=\frac{e^{a y-b x}}{2|\alpha| \sqrt{|\lambda|}} \leq \frac{1}{\sqrt{2|\lambda|}} .
$$

Case $x<0, y \geq 0$. In this case, the result follows from the previous step and the observation 3.2 .

Case $x<0, y<0$. In view of $(3.2)$, there is no loss of generality in assuming that $x \leq y$. Then elementary calculations show that

$$
\left|G_{\lambda}(x, y)\right|^{2}=\frac{1}{4|\lambda|} \Psi(x, y),
$$

where

$$
\Psi(x, y):=e^{-2 a(y-x)}+e^{2 a(y+x)}-2 e^{2 a x} \sin (2 b y) .
$$

In the same way as in the first case, by fixing $y \geq 0$ and considering $\Psi$ as a function of the variable $x$ on the interval $(-\infty, y]$, we come to the conclusion that

$$
\max _{x \leq y \leq 0} \Psi(x, y) \leq 2 \max \left(1+\frac{|\Re(\lambda)|}{|\lambda|}\right),
$$


completing the proof.

3.2. Krein resolvent formula. Let $B_{ \pm}=\mp \partial_{x}^{2}$ on $L^{2}\left(\mathbb{R}_{ \pm}\right)$with Dirichlet boundary conditions at the origin. We set

$$
B_{0}:=\left(\begin{array}{cc}
B_{+} & 0 \\
0 & B_{-}
\end{array}\right) \quad \text { on } \quad L^{2}(\mathbb{R})=L^{2}\left(\mathbb{R}_{+}\right) \oplus L^{2}\left(\mathbb{R}_{-}\right)
$$

and

$$
F_{\lambda} \psi:=\frac{1}{\mathrm{i}(\sqrt{\lambda}+\sqrt{-\lambda})}\left(J f_{\lambda}, \psi\right) f_{\lambda}
$$

where $J=\operatorname{sgn}(\cdot)$ and

$$
f_{\lambda}(x):=\mathrm{e}^{\mathrm{i} \sqrt{\lambda}} \mathbf{1}_{x>0}+\mathrm{e}^{-\mathrm{i} \sqrt{-\lambda}} \mathbf{1}_{x<0} .
$$

We use the following Krein type resolvent formula (see [5]):

$$
\left(H_{0}-\lambda\right)^{-1}=\left(B_{0}-\lambda\right)^{-1}-F_{\lambda} .
$$

The operator $F_{\lambda}$ has rank one, and its unique eigenvalue is given by

$$
\mu(\lambda)=\frac{1}{2 \mathrm{i}(\sqrt{\lambda}+\sqrt{-\lambda})}\left(\frac{1}{\Im(\sqrt{\lambda})}-\frac{1}{\Im(\sqrt{-\lambda})}\right) .
$$

We then have

$$
\left\|F_{\lambda}\right\|=\left\|F_{\lambda}\right\|_{\text {tr }}=|\mu(\lambda)| \leq \frac{1}{|\Im(\lambda)|},
$$

Together with the self-adjointness of $B_{0}$ and 3.18 this implies that

$$
\left\|\left(H_{0}-\lambda\right)^{-1}\right\| \leq \frac{2}{|\Im(\lambda)|} .
$$

\section{Limiting ABSORPtion TYPE PRINCIPLE}

The main role in the proofs of Theorems 1 in is played by the Birman-Schwinger operator

$$
K_{\lambda}:=|V|^{1 / 2}\left(H_{0}-\lambda\right)^{-1} V_{1 / 2} \quad \text { with } \quad V_{1 / 2}:=|V|^{1 / 2} \operatorname{sgn}(V),
$$

where sgn $: \mathbb{C} \rightarrow \mathbb{C}$ is the complex signum function defined by $\operatorname{sgn}(z):=z /|z|$ for $z \neq 0$ with the convention $\operatorname{sgn}(0):=0$. The operator $K_{\lambda}$ is well defined on its natural domain of the composition of three operators for all $\lambda \in \mathbb{C}$. Furthermore, we have a useful formula for its integral kernel

$$
K_{\lambda}(x, y)=|V|^{1 / 2}(x) G_{\lambda}(x, y) V_{1 / 2}(y),
$$

where $G_{\lambda}$ is the Green's function of $H_{0}-\lambda$.

The following lemma can be considered as a one-sided version of the conventional BirmanSchwinger principle extended to possibly embedded eigenvalues. Its proof is heavily inspired by the ones of the analogous results in $25,30,32$.

Lemma 2. Assume that $V \in L^{1}(\mathbb{R})$. Let $\lambda \in \sigma_{\mathrm{p}}\left(H_{V}\right) \cap\left(\mathbb{C}^{+} \cup \mathbb{R}\right)$ and $\psi \in \mathrm{D}\left(H_{V}\right)$ be an associated eigenvector. Then $\phi:=|V|^{1 / 2} \psi \in L^{2}(\mathbb{R})$ and

$$
\forall \varphi \in L^{2}(\mathbb{R}), \quad \lim _{\varepsilon \rightarrow 0^{+}}\left(\varphi, K_{\lambda+i \varepsilon} \phi\right)=-(\varphi, \phi) .
$$

In particular,

$$
\liminf _{\varepsilon \rightarrow 0^{+}}\left\|K_{\lambda+i \varepsilon}\right\| \geq 1
$$


Proof. It readily follows from 1.3 that $\phi \in L^{2}(\mathbb{R})$. We fix $\varphi \in L^{2}(\mathbb{R})$. Given any $\lambda \in$ $\sigma_{\mathrm{p}}\left(H_{V}\right)$, we have $z:=\lambda+i \varepsilon \in \mathbb{C}^{+} \subset \rho\left(H_{0}\right)$ for all $\varepsilon>0$. Furthermore,

$$
\begin{aligned}
\left(\varphi, K_{z} \phi\right) & =\iint_{\mathbb{R} \times \mathbb{R}} \overline{\varphi(x)}|V|^{1 / 2}(x) G_{z}(x, y) V(y) \psi(y) \mathrm{d} x \mathrm{~d} y \\
& =\int_{\mathbb{R}} \eta_{\varepsilon}(y) V(y) \psi(y) \operatorname{sgn}(y) \mathrm{d} y
\end{aligned}
$$

where

$$
\eta_{\varepsilon}:=\operatorname{sgn}(\cdot)\left(\int_{\mathbb{R}} \overline{\varphi(x)}|V|^{1 / 2}(x) G_{z}(x, \cdot) \mathrm{d} x\right)=\left(H_{0}-z\right)^{-1}|V|^{1 / 2} \bar{\varphi} \operatorname{sgn} .
$$

Here the second equality holds due to the (anti-)symmetric property of the Green's function in (3.1): $G_{z}(x, y)=G_{z}(y, x)$ if $\operatorname{sgn}(x)=\operatorname{sgn}(y)$ and $G_{z}(x, y)=-G_{z}(y, x)$ if $\operatorname{sgn}(x)=$ $-\operatorname{sgn}(y)$. By the Cauchy-Schwarz inequality, $|V|^{1 / 2} \bar{\varphi} \in L^{2}(\mathbb{R})$. Since $z \notin \sigma\left(H_{0}\right)$, we have $\eta_{\varepsilon} \in \mathrm{D}\left(H_{0}\right) \in H^{1}(\mathbb{R})$, and the weak formulation of the eigenvalue equation $H_{V} \psi=\lambda \psi$ yields

$$
\begin{aligned}
\int_{\mathbb{R}} \eta_{\varepsilon}(y) V(y) \psi(y) \operatorname{sgn}(y) \mathrm{d} y & =-\left({\overline{\eta_{\varepsilon}}}^{\prime}, \psi^{\prime}\right)+\lambda\left(\overline{\eta_{\varepsilon}}, \psi \operatorname{sgn}\right) \\
& =-\left(\bar{\psi}^{\prime}, \eta_{\varepsilon}^{\prime}\right)+\lambda\left(\bar{\psi} \operatorname{sgn}, \eta_{\varepsilon}\right) \\
& =-\left(\bar{\psi}^{\prime}, \eta_{\varepsilon}^{\prime}\right)+z\left(\bar{\psi} \operatorname{sgn}, \eta_{\varepsilon}\right)-i \varepsilon\left(\bar{\psi} \operatorname{sgn}, \eta_{\varepsilon}\right) \\
& =-\left(\bar{\psi},|V|^{1 / 2} \bar{\varphi}\right)-i \varepsilon\left(\bar{\psi} \operatorname{sgn}, \eta_{\varepsilon}\right) \\
& =-\left(\varphi,|V|^{1 / 2} \psi\right)-i \varepsilon\left(\overline{\eta_{\varepsilon}}, \psi \operatorname{sgn}\right) .
\end{aligned}
$$

Here the penultimate equality follows from the weak formulation of the resolvent equation $\left(H_{0}-z\right) \eta_{\varepsilon}=|V|^{1 / 2} \bar{\varphi}$ sgn. Consequently, (4.5) and (4.6) imply (4.3) after taking the limit $\varepsilon \rightarrow 0^{+}$, provided that $\varepsilon\left(\bar{\eta}_{\varepsilon}, \psi \operatorname{sgn}\right) \rightarrow 0$ as $\varepsilon \rightarrow 0^{+}$. To see the latter, we write

$$
\left|\left(\overline{\eta_{\varepsilon}}, \psi \operatorname{sgn}\right)\right|=\left|\left(\varphi \operatorname{sgn}, M_{\varepsilon} \psi \operatorname{sgn}\right)\right| \leq\|\varphi\|\left\|M_{\varepsilon}\right\|\|\psi\|,
$$

where $M_{\varepsilon}:=|V|^{1 / 2}\left(H_{0}-z\right)^{-1}$, and it remains to be shown that $\varepsilon\left\|M_{\varepsilon}\right\|$ tends to zero as $\varepsilon \rightarrow 0^{+}$. To this end, first we notice that

$$
\begin{aligned}
\sup _{x \geq 0} \int_{\mathbb{R}}\left|G_{z}(x, y)\right|^{2} d y & \leq \sup _{x \geq 0} \int_{-\infty}^{0}\left|G_{z}(x, y)\right|^{2} d y+\sup _{x \geq 0} \int_{0}^{\infty}\left|G_{z}(x, y)\right|^{2} \mathrm{~d} y \\
& \leq \frac{1}{2|z|} \int_{-\infty}^{0} e^{2 y \Re(\sqrt{z})} \mathrm{d} y+\frac{1}{|z|} \int_{0}^{\infty} e^{-2 y \Im(\sqrt{z})} \mathrm{d} y \\
& =\frac{1}{4|z|}\left[\frac{1}{\Re(\sqrt{z})}+\frac{2}{\Im(\sqrt{z})}\right]
\end{aligned}
$$

and that

$$
\begin{aligned}
\sup _{x \leq 0} \int_{\mathbb{R}}\left|G_{z}(x, y)\right|^{2} \mathrm{~d} y & \leq \sup _{x \leq 0} \int_{-\infty}^{0}\left|G_{z}(x, y)\right|^{2} \mathrm{~d} y+\sup _{x \leq 0} \int_{0}^{\infty}\left|G_{z}(x, y)\right|^{2} \mathrm{~d} y \\
& \leq \frac{1}{|z|} \int_{-\infty}^{0} e^{2 y \Re(\sqrt{z})} \mathrm{d} y+\frac{1}{2|z|} \int_{0}^{\infty} e^{-2 y \Im(\sqrt{z})} \mathrm{d} y \\
& =\frac{1}{4|z|}\left[\frac{2}{\Re(\sqrt{z})}+\frac{1}{\Im(\sqrt{z})}\right] .
\end{aligned}
$$


Therefore, it follows that

$$
\begin{aligned}
\left\|M_{\varepsilon}\right\|^{2} \leq\left\|M_{\varepsilon}\right\|_{\mathrm{HS}}^{2} & =\iint_{\mathbb{R} \times \mathbb{R}}|V(x)|\left|G_{z}(x, y)\right|^{2} \mathrm{~d} x \mathrm{~d} y \\
& \leq \sup _{x \in \mathbb{R}} \int_{\mathbb{R}}\left|G_{z}(x, y)\right|^{2} \mathrm{~d} y \int_{\mathbb{R}}|V(x)| \mathrm{d} x \\
& \leq \frac{1}{2|z|}\left[\frac{1}{\Re(\sqrt{z})}+\frac{1}{\Im(\sqrt{z})}\right] \int_{\mathbb{R}}|V(x)| \mathrm{d} x .
\end{aligned}
$$

On the other hand, elementary calculations show that

while

$$
|z| \Re(\sqrt{z}) \sim \begin{cases}\varepsilon^{1 / 2} & \text { if } \lambda=0, \\ 1 & \text { otherwise }\end{cases}
$$

$$
|z| \Im(\sqrt{z}) \sim \begin{cases}\varepsilon^{1 / 2} & \text { if } \lambda=0, \\ \varepsilon & \text { if } \Re(\lambda)>0 \& \Im(\lambda)=0, \\ 1 & \text { otherwise }\end{cases}
$$

Hence, we have $\left\|M_{\varepsilon}\right\|=\mathcal{O}\left(|\varepsilon|^{-3 / 4}\right)$ as $\varepsilon \rightarrow 0^{+}$, which concludes the proof of the claim in 4.3.

Now applying (4.3) with $\varphi=\phi$ and taking the limit $\varepsilon \rightarrow 0^{+}$, we obtain obtain

$$
|(\phi, \phi)| \leq\left|\left(\phi, K_{\lambda+i \varepsilon} \phi\right)\right| \leq \liminf _{\varepsilon \rightarrow 0^{+}}\left\|K_{\lambda+i \varepsilon}\right\|\|\phi\|^{2} .
$$

Since $\phi=|V|^{1 / 2} \psi \neq 0$ (if this were not true, then $\lambda$ would be an eigenvalue for $H_{0}$, unless $\psi=0$, which is impossible), we thus conclude 4.4.

\section{Proofs of the MAIN RESUlts}

Without loss of generality we work with fixed $\lambda \in \mathbb{C}^{+} \cup \mathbb{R}$ in the sequel. The key strategy of the proof is to estimate the norm the Birman-Schwinger operator from above and apply Lemma 2 .

5.1. Proof of Theorem 1. First, we consider the case $\lambda \in \mathbb{C}^{+}$. In view of Lemma 1, we can estimate the norm of the Birman-Schwinger operator as follows

$$
\begin{aligned}
\left\|K_{\lambda}\right\|^{2} \leq\left\|K_{\lambda}\right\|_{\mathrm{HS}}^{2} & =\iint_{\mathbb{R} \times \mathbb{R}}|V(x)|\left|G_{\lambda}(x, y)\right|^{2}|V(y)| \mathrm{d} x \mathrm{~d} y \\
& \leq \frac{1}{2}\left(\frac{1}{|\lambda|}+\frac{|\Re(\lambda)|}{|\lambda|^{2}}\right)\|V\|_{1}^{2} .
\end{aligned}
$$

If $\lambda \in \mathbb{R} \backslash\{0\}$, then the same analysis applied for $\lambda+i \varepsilon$ with $\varepsilon>0$ (instead of $\lambda$ ) yields

$$
\begin{aligned}
\liminf _{\varepsilon \rightarrow 0^{+}}\left\|K_{\lambda+i \varepsilon}\right\|^{2} & \leq \liminf _{\varepsilon \rightarrow 0^{+}} \frac{1}{2}\left(\frac{1}{|\lambda+i \varepsilon|}+\frac{|\Re(\lambda)|}{|\lambda+i \varepsilon|^{2}}\right)\|V\|_{1}^{2} \\
& =\frac{1}{2}\left(\frac{1}{|\lambda|}+\frac{|\Re(\lambda)|}{|\lambda|^{2}}\right)\|V\|_{1}^{2} .
\end{aligned}
$$

Hence, Lemma 2 implies that if $\lambda \in\left(\mathbb{C}^{+} \cup \mathbb{R}\right) \backslash\{0\}$ is an eigenvalue for $H_{V}$, then we must have

$$
2 \leq\left(\frac{1}{|\lambda|}+\frac{|\Re(\lambda)|}{|\lambda|^{2}}\right)\|V\|_{1}^{2},
$$

i.e. (1.4) must hold. This completes the proof since (1.4) trivially holds for $\lambda=0$. 
5.2. Optimality of the eigenvalue bound of Theorem 1. Here we demonstrate that the result (1.4) is sharp in the sense that to any non-real boundary point of the spectral enclosure, there exists a delta-potential $V$ so that this boundary point is an eigenvalue of $H_{V}$. By standard approximation arguments, it follows that there exists a sequence of $L^{1}$-potentials $V_{n}$ such that the eigenvalues of $H_{V_{n}}$ converge to those of $H_{V}$. This shows that that the boundary curve in 1.4 cannot be improved.

Let us take an arbitrary non-real boundary point $\lambda$ of the spectral enclosure. Since the boundary curve is symmetric with respect to the real axis, there is no loss of generality in assuming that $\lambda \in \mathbb{C}^{+}$. Let us denote the positive numbers $\Re(\sqrt{\lambda})$ and $\Im(\sqrt{\lambda})$ by $a$ and $b$, respectively. Further, for given $Q>0$, let us consider $\alpha \in \mathbb{C}$ with $|\alpha|=Q$ and the operator $H_{V}$ with the Dirac delta potential $V(x)=\alpha \delta\left(x-x_{0}\right), x \in \mathbb{R}$, where

$$
x_{0}=\frac{1}{2 a} \arccos \left(\frac{2 a b}{a^{2}+b^{2}}\right) .
$$

The operator $H_{V}$ can be defined rigorously by form methods (see Section 2). In this case, the Birman-Schwinger operator reduces to the multiplication operator with the constant function $\alpha G_{\lambda}\left(x_{0}, x_{0}\right)$, where $G_{\lambda}$ is the Green's function defined in (3.1), and the inequality (5.1) becomes equality. Furthermore, we have $Q\left|G_{\lambda}\left(x_{0}, x_{0}\right)\right|=1$ (see the proof of Lemma 1 ). Hence, by fixing the phase of $\alpha$ in such a way that $\alpha G_{\lambda}\left(x_{0}, x_{0}\right)=1$, we deduce from the Birman-Schwinger principle that $\lambda \in \sigma\left(H_{V}\right)$. On the other hand, we have $\sigma_{\text {ess }}\left(H_{V}\right)=\mathbb{R}$ (since the perturbation is a point interaction) and $\sigma_{\mathrm{r}}\left(H_{V}\right)=\emptyset$ (since $H_{V}$ is $J$-self-adjoint, where $J$ is the complex conjugation operator). Therefore, $\lambda \in \mathbb{C}^{+}$must be a discrete eigenvalue for $H_{V}$.

5.3. Proof of Theorem 2. The proof uses complex interpolation and the result of Theorem 1. Observe that (1.6) holds trivially for $\lambda \in \mathbb{R}$. For $\lambda \in \mathbb{C}^{+}$, let us consider the operator family

$$
T_{z}:=|V|^{z p / 2}\left(H_{0}-\lambda\right)^{-1}|V|^{z p / 2},
$$

for $z \in \mathbb{C}$ with $0 \leq \Re z \leq 1$. First, we note that $T_{1}$ is a bounded operator under our hypothesis on $V$. Indeed, $|V|^{p / 2}$ maps $L^{2}(\mathbb{R})$ to $H^{-1}(\mathbb{R})$ by duality and $\left(H_{0}-\lambda\right)^{-1}$ is an isomorphism between $H^{-1}(\mathbb{R})$ and $H^{1}(\mathbb{R})$, while the latter space is mapped by $|V|^{p / 2}$ back to $L^{2}(\mathbb{R})$. Further, we note that $T_{z}$ is continuous in the closed strip $0 \leq \Re z \leq 1$, analytic in its interior and we have

$$
\sup _{0 \leq \Re z \leq 1}\left\|T_{z}\right\| \leq \max \left\{\frac{2}{|\Im(\lambda)|},\left\|T_{1}\right\|\right\},
$$

see (3.19). In particular, $T_{z}$ is uniformly bounded for $0 \leq \Re z \leq 1$. Since $V \in L^{p}(\mathbb{R})$ by the hypothesis, we can proceed as in the proof of Theorem 1 and conclude

$$
\left\|T_{1+\mathrm{i} y}\right\| \leq\left\||V|^{p / 2}\left(H_{0}-\lambda\right)^{-1}|V|^{p / 2}\right\| \leq \frac{\sqrt{|\lambda|+|\Re(\lambda)|}}{\sqrt{2}|\lambda|}\|V\|_{p}^{p},
$$

for any $y \in \mathbb{R}$. Moreover, for all $y \in \mathbb{R}$, we have also the trivial estimate

$$
\left\|T_{\mathrm{i} y}\right\| \leq \frac{2}{|\Im(\lambda)|},
$$

see (3.19). Thus, Stein's complex interpolation theorem (see e.g. [43, Thm. V.4.1]) yields the following bound for the Birman-Schwinger operator

$$
\|K(\lambda)\| \leq\left\|T_{1 / p}\right\| \leq \frac{(|\lambda|+|\Re(\lambda)|)^{\frac{1}{2 p}}}{2^{\frac{3}{2 p}-1}|\lambda|^{\frac{1}{p}}} \frac{\|V\|_{p}}{|\Im(\lambda)|^{1-\frac{1}{p}}} .
$$


If $\lambda$ is an eigenvalue for $H_{V}$, then the standard Birman-Schwinger principle implies that the expression on the right-hand-side of (5.3) cannot be strictly less than 1, thus yielding the estimate (1.6).

5.4. Proofs of Corollaries 1 and 2, Let $1 \leq p<\infty$. For $\lambda \in \mathbb{C}^{+}$, let $x=\Re(\lambda)$ and $y=\Im(\lambda)$. Then (1.6) (resp. (1.4) ) can be written, equivalently, as

$$
2^{3-2 p}\left(x^{2}+y^{2}\right) y^{2 p-2} \leq\left(\sqrt{x^{2}+y^{2}}+|x|\right)\|V\|_{p}^{2 p} .
$$

Since the region corresponding to $\sqrt{5.4}$ is symmetric with respect to the imaginary axis, there is no loss of generality in assuming that $x \geq 0$. Further with the change of the variables $x=\|V\|_{p}^{\frac{2 p}{2 p-1}} t, y=\|V\|_{p}^{\frac{2 p}{2 p-1}} s$, (5.4) reads as

$$
2^{3-2 p}\left(t^{2}+s^{2}\right) s^{2 p-2} \leq \sqrt{t^{2}+s^{2}}+t .
$$

Next, let us consider the function

$$
f(x)=\frac{x}{1+x^{2}}+\frac{x}{\sqrt{1+x^{2}}}
$$

for $x \geq 0$. Letting $x=\tan (\alpha)$ for $\alpha \in\left[0, \frac{\pi}{2}\right)$, we easily get $f(\tan (\alpha))=\sin (\alpha)+\frac{1}{2} \sin (2 \alpha)$ which attains its global maximum at $\alpha=\frac{\pi}{3}$. Consequently, we have

$$
\sup _{x \geq 0} f(x)=\sup _{\alpha \in\left[0, \frac{\pi}{2}\right]} f(\tan (\alpha))=\frac{3 \sqrt{3}}{4}=f(\sqrt{3}) .
$$

Hence, it follows from 5.5 that $2^{3-2 p} s^{2 p-1} \leq f(s / t) \leq 3 \sqrt{3} / 4$ for all $s \geq 0$ and $t>0$. Therefore,

$$
s \leq 2\left(\frac{3 \sqrt{3}}{16}\right)^{\frac{1}{2 p-1}}
$$

and 5.5 becomes equality if and only if

$$
(s, t)=\left(2\left(\frac{3 \sqrt{3}}{16}\right)^{\frac{1}{2 p-1}}, \frac{2}{\sqrt{3}}\left(\frac{3 \sqrt{3}}{16}\right)^{\frac{1}{2 p-1}}\right),
$$

proving the claim.

5.5. Proof of Theorem 3. Let $\lambda \in \mathbb{C}^{+}$and denote by $\Omega$ the support of $V$. For arbitrary weight function $\rho>0$, the Schur test yields

$$
\|K(\lambda)\| \leq\left(\sup _{x \in \Omega} \int_{\Omega}\left|K_{\lambda}(x, y)\right| \frac{\mathrm{d} y}{\rho(x, y)}\right)^{1 / 2}\left(\sup _{y \in \Omega} \int_{\Omega}\left|K_{\lambda}(x, y)\right| \rho(x, y) \mathrm{d} x\right)^{1 / 2} .
$$

By choosing the weight function

$$
\rho(x, y):=|V(x)|^{1 / 2}|V(y)|^{-1 / 2}, \quad x, y \in \Omega,
$$

and using 3.2 , we obtain

$$
\|K(\lambda)\| \leq \sup _{x \in \Omega} \int_{\Omega}\left|G_{\lambda}(x, y)\right||V(y)| \mathrm{d} y \leq \sup _{x \in \mathbb{R}} \int_{\mathbb{R}}\left|G_{\lambda}(x, y)\right||V(y)| \mathrm{d} y .
$$

On the other hand, in view of $(3.1)$, we have

$$
\begin{aligned}
\sup _{x \geq 0} \int_{\mathbb{R}}\left|G_{\lambda}(x, y)\right||V(y)| \mathrm{d} y & \leq \sup _{x \geq 0} \int_{-\infty}^{0}\left|G_{\lambda}(x, y)\right||V(y)| \mathrm{d} y+\sup _{x \geq 0} \int_{0}^{\infty}\left|G_{\lambda}(x, y)\right||V(y)| \mathrm{d} y \\
& \leq \frac{1}{\sqrt{2|\lambda|}} \int_{-\infty}^{0} e^{\Re(\sqrt{\lambda}) y}|V(y)| \mathrm{d} y+\frac{1}{\sqrt{|\lambda|}} \int_{0}^{\infty} e^{-\Im(\sqrt{\lambda}) y}|V(y)| \mathrm{d} y .
\end{aligned}
$$


By the Hölder inequality,

$$
\int_{-\infty}^{0} e^{\Re(\sqrt{\lambda}) y}|V(y)| \mathrm{d} y \leq\|V\|_{p,-}\left(\int_{-\infty}^{0} e^{q \Re(\sqrt{\lambda}) y} \mathrm{~d} y\right)^{1 / q}=\frac{\|V\|_{p,-}}{\sqrt[q]{q \Re(\sqrt{\lambda})}}
$$

and, similarly,

$$
\int_{0}^{\infty} e^{-\Im(\sqrt{\lambda}) y}|V(y)| \mathrm{d} y \leq \frac{\|V\|_{p,+}}{\sqrt[q]{q \Im(\sqrt{\lambda})}}
$$

Therefore, we have

$$
\sup _{x \geq 0} \int_{\mathbb{R}}\left|G_{\lambda}(x, y)\right||V(y)| \mathrm{d} y \leq \frac{1}{\sqrt[q]{q} \sqrt{|\lambda|}}\left(\frac{\|V\|_{p,-}}{\sqrt{2} \sqrt[q]{\Re(\sqrt{\lambda})}}+\frac{\|V\|_{p,+}}{\sqrt[q]{\Im(\sqrt{\lambda})}}\right)
$$

and, analogously,

$$
\sup _{x \leq 0} \int_{\mathbb{R}}\left|G_{\lambda}(x, y)\right||V(y)| \mathrm{d} y \leq \frac{1}{\sqrt[q]{q} \sqrt{|\lambda|}}\left(\frac{\|V\|_{p,-}}{\sqrt[q]{\Re(\sqrt{\lambda})}}+\frac{\|V\|_{p,+}}{\sqrt{2} \sqrt[q]{\Im(\sqrt{\lambda})}}\right) .
$$

Recalling (5.8), we conclude that the maximum of the quantities on the right-hand-sides of (5.9) and (5.10) dominates the operator norm of the Birman-Schwinger operator $K(\lambda)$ and the result immediately follows from the Birman-Schwinger principle as in the proof of Theorem 2 .

5.6. Proof of Theorem 4. First we establish an auxiliary lemma. The proof is a straightforward calculation and is omitted.

Lemma 3. Let $\lambda>0$ be arbitrary. Then the function

$$
u(x)=\mathrm{e}^{\sqrt{\lambda} x} \mathbf{1}_{x \leq 0}+\sqrt{2} \sin (\sqrt{\lambda} x+\pi / 4) \mathbf{1}_{x \geq 0}
$$

satisfies $H_{0} u=\lambda u$ in the weak sense. Moreover, $u \in L^{2}\left(\mathbb{R}_{-}\right)$and $u \notin L^{2}\left(\mathbb{R}_{+}\right)$.

Proof of Theorem 4. We first discuss the case $n=1$. Set $\psi(x)=u(x) \chi(x)$, with $u$ given by (5.11) and with $\chi \in C^{\infty}(\mathbb{R})$ to be chosen later. Then

$$
\left(H_{0}-\lambda\right) \psi(x)=-\operatorname{sgn}(x)\left(2 u^{\prime}(x) \chi^{\prime}(x)+u(x) \chi^{\prime \prime}(x)\right) .
$$

Selecting

$$
V=2 \frac{u^{\prime} \chi^{\prime}}{u \chi}+\frac{\chi^{\prime \prime}}{\chi}
$$

the equation $H_{V} \psi=\lambda \psi$ is satisfied by definition, provided that $V$ is well-defined. The issue is of course that $u$ has zeros on $\mathbb{R}_{+}$. To cancel these we first set

$$
g(x)=\int_{0}^{x} \sin ^{2}(\sqrt{\lambda} t+\pi / 4) \mathrm{d} t, \quad x \in \mathbb{R}
$$

and choose

$$
\chi(x)=\left(1+g(x)^{2}\right)^{-1}, \quad x \in \mathbb{R} .
$$

We then get

$$
V=\frac{8 g^{2} g^{\prime 2}}{\left(1+g^{2}\right)^{2}}-\frac{2\left(g^{2}+g g^{\prime \prime}\right)}{1+g^{2}}-\frac{4 g g^{\prime} u^{\prime}}{u\left(1+g^{2}\right)}
$$


Since $g^{\prime} / u$ vanishes on the zero set of $u$ and $g(x) \geq c|x|$ for some $c>0$ and all sufficiently large $x$, we see that $V$ satisfies 1.12 for $n=1$. For arbitrary $n \in \mathbb{N}$ we replace $\chi$ in (5.13) by $\left(n^{2}+g(x)^{2}\right)^{-1}$.

5.7. Proof of Theorem 5. By scaling (1.10) with $\rho=\sqrt{\mu}$ we may assume that $\mu=1$. We make the following Ansatz for the wavefunction $\psi$ :

$$
\psi(x)= \begin{cases}\mathrm{e}^{\sqrt{\lambda} x} & x \leq 0, \\ A \mathrm{e}^{\mathrm{i} k x}+B \mathrm{e}^{-\mathrm{i} k x} & 0 \leq x \leq R, \\ C \mathrm{e}^{\mathrm{i} \sqrt{\lambda} x} & x \geq R,\end{cases}
$$

where

$$
k^{2}+V_{0}=\lambda, \quad \Im(k)>0, \quad \Im(\sqrt{\lambda})>0, \quad \Re(\sqrt{\lambda})>0
$$

and $R=R(\varepsilon), k=k(\varepsilon)$ will be chosen later. Taking

$$
A=A(k, \lambda)=\frac{1}{2}+\frac{\sqrt{\lambda}}{2 \mathrm{i} k}, \quad B=B(k, \lambda)=\frac{1}{2}-\frac{\sqrt{\lambda}}{2 \mathrm{i} k},
$$

it follows that $\psi, \psi^{\prime}$ are continuous at $x=0$. It is easy to see that there exists $C \in \mathbb{C}$ such that $\psi, \psi^{\prime}$ are continuous at $x=R$ if and only if

$$
\sqrt{\lambda}=-k \frac{B-A \mathrm{e}^{2 \mathrm{i} k R}}{B+A \mathrm{e}^{2 \mathrm{i} k R}} .
$$

We set

$$
k=k(\varepsilon)=-1+\mathrm{i} \varepsilon, \quad R=R(\varepsilon)=\frac{|\ln \varepsilon|}{2 \varepsilon}+\theta
$$

with $\theta=\theta(\varepsilon) \in[0, \pi]$ to be chosen later. Changing variables from $\lambda$ to $\omega$ v.i.z. $\sqrt{\lambda}=1+\mathrm{i} \varepsilon+\omega$ and setting

$$
f_{\varepsilon}(\omega)=1+\mathrm{i} \varepsilon+\omega+k \frac{B-A \mathrm{e}^{2 \mathrm{i} k R}}{B+A \mathrm{e}^{2 \mathrm{i} k R}}, \quad \omega \in B\left(0, C \varepsilon^{2}\right),
$$

with $C$ independent of $\varepsilon$ to be chosen sufficiently large, we see that $(5.15)$ is equivalent to $f_{\varepsilon}(\omega)=0$. Since $\mathrm{e}^{-2 \operatorname{Im} k R}=\varepsilon(1+\mathcal{O}(\varepsilon))$ and $A / B=(-1+\mathrm{i}) /(1+\mathrm{i})+\mathcal{O}(\varepsilon)$ as $\varepsilon \rightarrow 0^{+}$, we obtain, by choosing $\theta(\varepsilon)$ such that $(-1+\mathrm{i}) /(1+\mathrm{i}) \mathrm{e}^{-2 \mathrm{i} R(\varepsilon)}=-1$,

$$
\begin{aligned}
\left|f_{\varepsilon}(\omega)-\omega\right| & =\left|1+\mathrm{i} \varepsilon+(-1+\mathrm{i} \varepsilon)\left(1-2 A / B \mathrm{e}^{2 \mathrm{i} k R}+\mathcal{O}\left(\varepsilon^{2}\right)\right)\right| \\
& =\left|2 \mathrm{i} \varepsilon+2 A / B \varepsilon \mathrm{e}^{-2 \mathrm{i} R}+\mathcal{O}\left(\varepsilon^{2}\right)\right|=\mathcal{O}\left(\varepsilon^{2}\right)
\end{aligned}
$$

as $\varepsilon \rightarrow 0^{+}$. It follows that, for $\varepsilon$ sufficiently small and $C$ sufficiently large,

$$
\left|f_{\varepsilon}(\omega)-\omega\right|<|\omega|, \quad \omega \in \partial B\left(0, C \varepsilon^{2}\right) .
$$

By Rouché's theorem $f_{\varepsilon}$ has exactly one zero in $B\left(0, C \varepsilon^{2}\right)$. This implies the existence of an eigenvalue $\lambda$ with the claimed properties. Recalling the relation between $k$ and $V_{0}$ in (5.14) and using 5.16 we get the estimate

$$
\|V(\varepsilon)\|_{p} \approx \varepsilon R(\varepsilon)^{1 / p}=\mathcal{O}\left(\varepsilon^{1-1 / p}|\ln \varepsilon|^{1 / p}\right)
$$

as $\varepsilon \rightarrow 0^{+}$. This proves 1.13.

Sharpness of exponents in (1.6): Since $|\lambda| \approx|\Re(\lambda)| \approx 1$ in the above example, in equality (1.6) says that

$$
\varepsilon^{1-1 / p} \leq C\|V(\varepsilon)\|_{p} .
$$


Comparing this to (5.17) we see that this is essentially sharp (up to logarithms). In particular, the exponent $1-1 / p$ of $\varepsilon \approx \Im(\lambda)$ cannot be made smaller.

5.8. Proof of Theorem 6. As usual, we split the Birman-Schwinger operator into a singular and a regular part (as $\lambda \rightarrow 0)$,

$$
K_{\lambda}=L_{\lambda}+M_{\lambda}
$$

We first ignore $M_{\lambda}$ and concentrate on $L_{\lambda}$,

$$
\begin{aligned}
L_{\lambda} \psi & =\frac{1}{2 \alpha \sqrt{\lambda}}\left(\left(g^{+}, \psi\right) f^{+}-\left(g^{-}, \psi\right) f^{+}+\left(g^{+}, \psi\right) f^{-}-\left(g^{-}, \psi\right) f^{-}\right) \\
& =\frac{1}{2 \alpha \sqrt{\lambda}}\left(g^{+}-g^{-}, \psi\right)|V|^{1 / 2}
\end{aligned}
$$

where we set

$$
f^{ \pm}(x)=\mathbf{1}_{ \pm}(x)|V(x)|^{1 / 2}, \quad g^{ \pm}(x)=\mathbf{1}_{ \pm}(x) V_{1 / 2}(x), \quad \mathbf{1}_{ \pm}=\mathbf{1}_{\mathbb{R}_{ \pm}} .
$$

From the second equality in 5.18 we see that $L_{\lambda}$ has rank 1 ; then

$$
\operatorname{det}\left(I+\varepsilon L_{\lambda}\right)=1+\varepsilon \operatorname{Tr} L_{\lambda}=1+\frac{\varepsilon}{2 \alpha \sqrt{\lambda}} v_{\mathrm{sgn}}
$$

Hence,

$$
0 \in \sigma\left(I+\varepsilon L_{\lambda}\right) \Longleftrightarrow \operatorname{det}\left(I+\varepsilon L_{\lambda}\right)=0 \Longleftrightarrow-\sqrt{\lambda}=\frac{\varepsilon}{2 \alpha} v_{\text {sgn }} .
$$

Since we are assuming that $\Im(\sqrt{\lambda})>0$ the rightmost equality can only hold if $\Re\left(v_{\text {sgn }}\right)+$ $\Im\left(v_{\text {sgn }}\right)<0$. We also see that

$$
\lambda \in \mathbb{C}^{+} \Longleftrightarrow \Re(\sqrt{\lambda})>0 \Longleftrightarrow \Re\left(v_{\text {sgn }}\right)<\Im\left(v_{\text {sgn }}\right) .
$$

We now repeat the argument, but this time taking $M_{\lambda}$ into account. The key observation is that the function $(x, y) \mapsto|\lambda|\left|M_{\lambda}(x, y)\right|^{2}$ is bounded from above, up to a constant, by the $L^{1}$-majorant $(x, y) \mapsto|V(x)||V(y)|$. Clearly,

$$
\lim _{\lambda \rightarrow 0}|\lambda|\left|M_{\lambda}(x, y)\right|^{2}=0 .
$$

Hence, by dominated convergence,

$$
\left\|M_{\lambda}\right\|_{\mathrm{HS}}=o\left(|\lambda|^{-1 / 2}\right), \quad(\lambda \rightarrow 0) .
$$

Since we expect that $c^{-1} \varepsilon \leq|\lambda|^{1 / 2} \leq c \varepsilon$ for $\lambda=\lambda(\varepsilon)$ and some $c>0$, we assume this from now on; we will see later that this assumption is indeed justified. Since then $\varepsilon\left\|M_{\lambda}\right\|<1$ for sufficiently small $\varepsilon$, it follows that $\left(I+\varepsilon M_{\lambda}\right)$ is invertible and

$$
0 \in \sigma\left(I+\varepsilon K_{\lambda}\right) \Longleftrightarrow \operatorname{det}\left(I+\varepsilon\left(I+\varepsilon M_{\lambda}\right)^{-1} L_{\lambda}\right)=0 .
$$

Similarly as before,

$$
\begin{aligned}
\operatorname{det}\left(I+\varepsilon\left(I+\varepsilon M_{\lambda}\right)^{-1} L_{\lambda}\right) & =1+\varepsilon \operatorname{Tr}\left(I+\varepsilon M_{\lambda}\right)^{-1} L_{\lambda} \\
& =1+\frac{\varepsilon}{2 \alpha \sqrt{\lambda}} v_{\mathrm{sgn}}+r(\varepsilon, \lambda),
\end{aligned}
$$

where $r(\varepsilon, \lambda)=O\left(\varepsilon\left\|M_{\lambda}\right\|\right)$. We now change variables from $\lambda$ to $z=\sqrt{\lambda}$. Clearly, $f_{\varepsilon}(z):=$ $\operatorname{det}\left(I+\varepsilon\left(I+\varepsilon M_{\lambda}\right)^{-1} L_{\lambda}\right)$ is an analytic function for $z \in B\left(-\frac{\varepsilon}{2 \alpha} v_{\mathrm{sgn}}, \rho(\varepsilon)\right)$, provided $\rho(\varepsilon)=$ $o(\varepsilon)$; we may choose $\rho(\varepsilon)=\max _{c^{-1} \varepsilon \leq|\lambda|^{1 / 2} \leq c \varepsilon}|r(\varepsilon, \lambda)|$. Then we have

$$
\left|f_{\varepsilon}(z)-\left(1+\frac{\varepsilon}{2 \alpha z} v_{\mathrm{sgn}}\right)\right| \leq \rho(\varepsilon), \quad z \in \partial B\left(-\frac{\varepsilon}{2 \alpha} v_{\mathrm{sgn}}, \rho(\varepsilon)\right) .
$$


On the other hand, since $v_{\mathrm{sgn}} \neq 0$, we have

$$
\left|1+\frac{\varepsilon}{2 \alpha z} v_{\mathrm{sgn}}\right| \geq \frac{2|\alpha| \rho(\varepsilon)}{\varepsilon\left|v_{\mathrm{sgn}}\right|+2|\alpha| \rho(\varepsilon)} \geq \frac{\rho(\varepsilon)}{\varepsilon\left|v_{\mathrm{sgn}}\right|}, \quad z \in \partial B\left(-\frac{\varepsilon}{2 \alpha} v_{\mathrm{sgn}}, \rho(\varepsilon)\right) .
$$

Hence, for $\varepsilon<1 /\left|v_{\mathrm{sgn}}\right|$, we have

$$
\left|f_{\varepsilon}(z)-\left(1+\frac{\varepsilon}{2 \alpha z} v_{\mathrm{sgn}}\right)\right|<\left|1+\frac{\varepsilon}{2 \alpha z} v_{\mathrm{sgn}}\right|, \quad z \in \partial B\left(-\frac{\varepsilon}{2 \alpha} v_{\mathrm{sgn}}, \rho(\varepsilon)\right) .
$$

By Rouché's theorem, $f_{\varepsilon}$ has exactly one zero in $B\left(-\frac{\varepsilon}{2 \alpha} v_{\mathrm{sgn}}, \rho(\varepsilon)\right)$. Since $\rho(\varepsilon)=o(\varepsilon)$ this proves the theorem.

Sharpness of (1.4): If we assume that $V$ is real-valued and $v_{\mathrm{sgn}}<0$, then the assumptions of Theorem 6 are satisfied. If we assume in addition that $V$ is supported either on $\mathbb{R}_{+}$or on $\mathbb{R}_{-}$, then $v_{\text {sgn }}=-\|V\|_{1}$. Since $1 / \alpha^{2}=2 \mathrm{i}$ we see that

$$
\lambda(\varepsilon)=\frac{\mathrm{i}}{2}\|\varepsilon V\|_{1}^{2}+o\left(\varepsilon^{2}\right), \quad \Re(\lambda(\varepsilon))=o\left(\varepsilon^{2}\right) .
$$

This shows that inequality (1.4) is saturated in the limit $\varepsilon \rightarrow 0$.

5.9. Proof of Theorem 7, We start with the Schatten bound

$$
\left\|W_{1}\left(H_{0}-\lambda\right)^{-1} W_{2}\right\|_{\mathfrak{S}^{2 p}} \leq C_{p}|\Im(\lambda)|^{1-\frac{1}{p}}|\lambda|^{-\frac{1}{2 p}}\left\|W_{1}\right\|_{2 p}\left\|W_{2}\right\|_{2 p}, \quad 1 \leq p \leq \infty,
$$

which is a consequence of $(3.19)$ and the pointwise bound for the resolvent kernel. Here and in the following we use the notation

$$
\|A\|_{\mathfrak{S}^{p}}^{p}:=\sum_{j} s_{j}(A)^{p}
$$

where $s_{j}(A)$ are the singular numbers of the compact operator $A$. We make the conformal transformation $z=\lambda^{2} \in \mathbb{C} \backslash[0, \infty)$ and apply Theorem 3.1 in $[27$ to the analytic family $z \mapsto K(\lambda(z))$, which by $(5.20)$ satisfies the bound

$$
\|K(\lambda(z))\|_{\mathfrak{S}^{2 p}} \leq C_{p}|\Im(\sqrt{z})|^{1-\frac{1}{p}}|z|^{-\frac{1}{4 p}}\|V\|_{p} .
$$

In particular, for $p=1$, we have

$$
\|K(\lambda(z))\|_{\mathfrak{S}^{2}} \leq C|z|^{-\frac{1}{4}}\|V\|_{p} .
$$

By [27, Theorem 3.1], for any $\varepsilon>0$, there exists $C$ such that

$$
\sum_{j} \delta\left(z_{j}\right)\left|z_{j}\right|^{-\frac{1}{2}+\frac{1}{2}\left(-\frac{1}{2}+\varepsilon\right)_{+}} \leq C\|V\|_{1}^{2\left(1+\left(-\frac{1}{2}+\varepsilon\right)_{+}\right)} .
$$

Taking $\varepsilon=1 / 2$ and using the distortion bound $|\Im(\lambda)| \approx \delta\left(z_{j}\right)\left|z_{j}\right|^{-\frac{1}{2}}$ yields the claim.

5.10. Proof of Theorem 8. We write $\lambda=k^{2}$ for the spectral parameter, where $k \in \mathbb{C} \backslash\{0\}$. This amounts to a double covering of the punctured complex $\lambda$-plane, with $\Im(k)<0$ corresponding to the second (unphysical) sheet. From 3.4 3.5 we have that

$$
|G(\lambda(k))|^{2} \leq \frac{1}{|\lambda|} \mathrm{e}^{2 \Im(k)-(|x|+|y|)},
$$

which implies

$$
\|K(\lambda(k))\|_{\mathfrak{S}^{2}} \leq \frac{1}{|k|} \int_{\mathbb{R}} \mathrm{e}^{2 \Im(k)-|x|}|V(x)| \mathrm{d} x .
$$

This is analogous to 29, Proposition 4.1], and the proof follows from the same arguments as in 29 . 


\section{ACKNOWLEDGEMENT}

The authors are grateful to Professor Gian-Michele Graf for stimulating discussions.

\section{REFERENCES}

[1] Abramov, A. A., Aslanyan, A., and Davies, E. B. Bounds on complex eigenvalues and resonances. J. Phys. A 34, 1 (2001), 57-72.

[2] Adams, R. A. Sobolev Spaces. Academic Press, New York, 1975.

[3] Behrndt, J. An open problem: accumulation of nonreal eigenvalues of indefinite Sturm-Liouville operators. Integral Equations Operator Theory 77, 3 (2013), 299-301.

[4] Behrndt, J., Katatbeh, Q., And Trunk, C. Non-real eigenvalues of singular indefinite Sturm-Liouville operators. Proc. Amer. Math. Soc. 137, 11 (2009), 3797-3806.

[5] Behrndt, J., Philipp, F., And Trunk, C. Bounds on the non-real spectrum of differential operators with indefinite weights. Math. Ann. 357, 1 (2013), 185-213.

[6] Behrndt, J., Schmitz, P., And Trunk, C. Estimates for the non-real spectrum of a singular indefinite Sturm-Liouville operator on $\mathbb{R}$. Proc. Appl. Math. Mech. 17 (2017), 859-860.

[7] Behrndt, J., Schmitz, P., And Trunk, C. Spectral bounds for singular indefinite Sturm-Liouville operators with $L^{1}$-potentials. Proc. Amer. Math. Soc. 146, 9 (2018), 3935-3942.

[8] Behrndt, J., Schmitz, P., And Trunk, C. Spectral bounds for indefinite singular Sturm-Liouville operators with uniformly locally integrable potentials. J. Differential Equations 267, 1 (2019), $468-493$.

[9] BöGLI, S. Schrödinger operator with non-zero accumulation points of complex eigenvalues. Comm. Math. Phys. 352, 2 (2017), 629-639.

[10] Cassanno, B., Ibrogimov, O. O., Krejčiřík, D., And Štampach, F. Location of eigenvalues of non-self-adjoint discrete Dirac operators. Ann. Henri Poincaré 21 (2020), 2193-2217.

[11] Cossetti, L. Bounds on eigenvalues of perturbed Lamé operators with complex potentials. Preprint, ArXiv: 1904. 08445v1[math. SP] (2019).

[12] Cuenin, J.-C. Eigenvalue bounds for Dirac and fractional Schrödinger operators with complex potentials. J. Funct. Anal. 272, 7 (2017), 2987-3018.

[13] Cuenin, J.-C. Improved eigenvalue bounds for Schrödinger operators with slowly decaying potentials. Commun. Math. Phys. 376 (2020), 2147-2160.

[14] Cuenin, J.-C., And Kenig, C. E. $L^{p}$ resolvent estimates for magnetic Schrödinger operators with unbounded background fields. Comm. Partial Differential Equations 42, 2 (2017), 235-260.

[15] Cuenin, J.-C., Laptev, A., And Tretter, C. Eigenvalue estimates for non-selfadjoint Dirac operators on the real line. Ann. Henri Poincaré 15, 4 (2014), 707-736.

[16] Cuenin, J.-C., And Siegl, P. Eigenvalues of one-dimensional non-self-adjoint Dirac operators and applications. Lett. Math. Phys. 108, 7 (2018), 1757-1778.

[17] Cuenin, J.-C., And Tretter, C. Non-symmetric perturbations of self-adjoint operators. J. Math. Anal. Appl. 441, 1 (2016), 235-258.

[18] Ćurgus, B., AND LAnger, H. A KreĬn space approach to symmetric ordinary differential operators with an indefinite weight function. J. Differential Equations 79, 1 (1989), 31-61.

[19] Davies, E. B., AND NATh, J. Schrödinger operators with slowly decaying potentials. J. Comput. Appl. Math. 148, 1 (2002), 1-28. On the occasion of the 65th birthday of Professor Michael Eastham.

[20] Demuth, M., Hansmann, M., and Katriel, G. On the discrete spectrum of non-selfadjoint operators. J. Funct. Anal. 257, 9 (2009), 2742-2759.

[21] Demuth, M., Hansmann, M., and Katriel, G. Eigenvalues of non-selfadjoint operators: a comparison of two approaches. In Mathematical physics, spectral theory and stochastic analysis, vol. 232 of Oper. Theory Adv. Appl. Birkhäuser/Springer Basel AG, Basel, 2013, pp. 107-163.

[22] Enblom, A. Estimates for eigenvalues of Schrödinger operators with complex-valued potentials. Lett. Math. Phys. 106, 2 (2016), 197-220.

[23] FAnelli, L., AND KREjČiŘík, D. Location of eigenvalues of three-dimensional non-self-adjoint Dirac operators. Lett. Math. Phys. (jan 2019).

[24] FAnelli, L., KREJČIŘíK, D., AND VEGA, L. Absence of eigenvalues of two-dimensional magnetic Schrödinger operators. J. Funct. Anal. 275, 9 (2018), 2453-2472.

[25] FAnelli, L., KREJČIŕí, D., And VEGA, L. Spectral stability of Schrödinger operators with subordinated complex potentials. J. Spectr. Theory 8, 2 (2018), 575-604.

[26] Frank, R. L. Eigenvalue bounds for Schrödinger operators with complex potentials. Bull. Lond. Math. Soc. 43, 4 (2011), 745-750. 
[27] Frank, R. L. Eigenvalue bounds for Schrödinger operators with complex potentials. III. Trans. Amer. Math. Soc. 370, 1 (2018), 219-240.

[28] Frank, R. L., Laptev, A., Lieb, E. H., And Seiringer, R. Lieb-Thirring inequalities for Schrödinger operators with complex-valued potentials. Lett. Math. Phys. 77, 3 (2006), 309-316.

[29] Frank, R. L., Laptev, A., And Safronov, O. On the number of eigenvalues of Schrödinger operators with complex potentials. J. Lond. Math. Soc. (2) 94, 2 (2016), 377-390.

[30] Frank, R. L., ANd Simon, B. Eigenvalue bounds for Schrödinger operators with complex potentials. II. J. Spectr. Theory 7, 3 (2017), 633-658.

[31] Henry, R., AND KREJČ́lŔíK, D. Pseudospectra of the Schrödinger operator with a discontinuous complex potential. J. Spectr. Theory 7, 3 (2017), 659-697.

[32] Ibrogimov, O. O., KrejČiŘík, D., and Laptev, A. Sharp bounds for eigenvalues of biharmonic operators with complex potentials in low dimensions. Preprint, ArXiv: 1903.01810v1 [math. SP] (2019).

[33] Ibrogimov, O. O., And Štampach, F. Spectral enclosures for non-self-adjoint discrete Schrödinger operators. Integral Equations Operator Theory 91, 6 (2019), Paper No. 53, 15.

[34] Karabash, I., And Trunk, C. Spectral properties of singular Sturm-Liouville operators with indefinite weight sgn $x$. Proc. Roy. Soc. Edinburgh Sect. A 139, 3 (2009), 483-503.

[35] Kato, T. Perturbation Theory for Linear Operators. Springer-Verlag, Berlin, 1966.

[36] KrejČiřík, D., And Siegl, P. Pseudomodes for Schrödinger operators with complex potentials. $J$. Funct. Anal. 276, 9 (2019), 2856-2900.

[37] Laptev, A., and Safronov, O. Eigenvalue estimates for Schrödinger operators with complex potentials. Comm. Math. Phys. 292, 1 (2009), 29-54.

[38] Lee, Y., And Seo, I. A note on eigenvalue bounds for Schrödinger operators. J. Math. Anal. Appl. 470, 1 (2019), 340-347.

[39] Levitin, M., And Seri, M. Accumulation of complex eigenvalues of an indefinite Sturm-Liouville operator with a shifted Coulomb potential. Oper. Matrices 10, 1 (2016), 223-245.

[40] Philipp, F. Relatively bounded perturbations of J-non-negative operators. Preprint, ArXiv: 1903. 03977v2 [math.SP] (2019).

[41] Reed, M., And Simon, B. Methods of Modern Mathematical Physics, II. Fourier Analysis. SelfAdjointness. Academic Press, New York, 1975.

[42] Safronov, O. Estimates for eigenvalues of the Schrödinger operator with a complex potential. Bull. Lond. Math. Soc. 42, 3 (2010), 452-456.

[43] Stein, E. M., And Weiss, G. Introduction to Fourier analysis on Euclidean spaces. Princeton University Press, Princeton, N.J., 1971. Princeton Mathematical Series, No. 32.

Department of Mathematical Sciences, Loughborough University, Loughborough, LeicesterShire, LE11 3TU United Kingdom

Email address: J.Cuenin@lboro.ac.uk

Institute for Theoretical Physics, ETh Zürich, Wolfgang-Pauli-Str. 27, 8093 Zürich, SwitzerLAND

Email address: oibrogimov@phys.ethz.ch 